\title{
A Geometric Approach for Distributed Multi-hop Target Localization in Cooperative Networks
}

\author{
Slavisa Tomic and Marko Beko
}

\begin{abstract}
This work addresses target localization problem in cooperative distributed sensor networks, in which all sensors are capable of measuring Received Signal Strength (RSS), but only some are appropriately equipped to measure Angle of Arrival (AOA) of the received signal. A novel approach based on simple geometry and multi-hopping is proposed, which allows for natural conversion of the problem into a Generalized Trust Region Sub-Problem (GTRS). The proposed algorithm comprises three main steps, each of them with linear computational cost in the number of neighbors, making it suitable for real-time applications. Our simulation results validate the performance of the new algorithm, surpassing some significantly more complex ones, and almost achieving a lower bound set by an existing algorithm which uses some (unrealistic) assumptions in its favor.
\end{abstract}

Index Terms-Distributed localization, Received Signal Strength (RSS), Angle Of Arrival (AOA), Weighted Central Mass (WCM), Generalized Trust Region Sub-Problem (GTRS).

\section{INTRODUCTION}

Fifth-Generation (5G) networks are expected to provide considerably higher bandwidth and faster data rates. This will enable connecting huge number of stationary and mobile devices (such as sensors, agents, users, machines, and vehicles) supporting Internet-of-Things, creating real-time dynamic networks of mobile things [1], [2], [3]. Hence, localization will gain additional importance, enabling deployment of new services and contributing to significantly improving the overall performance of the $5 \mathrm{G}$ system [1].

Localization based on range (through Received Signal Strength (RSS) or time of arrival) and/or bearing (Angle Of Arrival (AOA)) measurements has received much attention in the recent society lately [4]-[26]. Nonetheless, most of these approaches are designed for non-cooperative networks in which target/target communication is not allowed or require a central unit which gathers all measurements and performs all computations. This approach is not very suitable for largescale networks due to potential bottlenecks at and near the central unit, and because its computational complexity depends highly on the network size. In [20], a cooperative algorithm for vehicle localization was proposed. It uses intervehicle-distance measurements taken by a radio-ranging technique, as well as vehicle kinematics and road maps to estimate the relative location of vehicles in a cluster. The authors in [21] proposed

Copyright (c) 2015 IEEE. Personal use of this material is permitted. However, permission to use this material for any other purposes must be obtained from the IEEE by sending a request to pubs-permissions@ieee.org.

S. Tomic and M. Beko are with COPELABS, Universidade Lusófona de Humanidades e Tecnologias, Campo Grande 376, 1749 - 024 Lisboa, Portugal. (e-mails: slavisa.tomic@ulusofona.pt, beko.marko@ulusofona.pt). M. Beko is also with UNINOVA, Monte de Caparica, 2829-516 Caparica, Portugal. a probabilistic fingerprint approach to determine location of a mobile target in indoor environments, which significantly speeds-up computation without employing approximations. A tracking algorithm with asynchronous updates triggered by beacon packet receptions, from which AOA estimates are opportunistically obtained was presented in [22]. The authors in [22] combined the potential of antenna array processing with a suitably-designed cooperation strategy and exploited vehicle-to-vehicle and vehicle-to-infrastructure communications. In [23], the authors proposed an outdoor place of interest recognition algorithm, aimed at recognizing big outdoor places only by opportunistically exploiting Wireless Fidelity (WiFi) access points and mobile devices such as smartphones. They also introduced the concept of a spot and proposed an enhanced WiFi fingerprinting algorithm. A hybrid RSS/AOA localization scheme for distributed networks based on SecondOrder Cone Programming (SOCP) was proposed in [24]. In addition to sensors' locations, the authors in [24] considered their transmit powers as unknowns as well. Although the SOCP algorithm showed good localization accuracy, its computational complexity is elevated, qualifying it as impractical for realtime applications. Another recently proposed scheme is the Distributed Linear Least Squares (DLLS) in [25], where the authors assumed that all sensors are able to measure both RSS and AOA quantities of the received signal. First, the authors in [25] estimate unbiased distances by assuming that the noise powers are available. Afterwards, they take advantage of the distance estimates to, jointly with AOA measurements, form lines in such a manner that, when they are fixed at a known reference point at one end, the other end indicates an estimate of the target of interest. Hence, stacking the line equations in vectors resulted in DLLS. In [26], a Message Passing Hybrid Localization (MPHL) for cooperative distributed localization using joint distance and direction measurements was proposed. The idea was to provide a method for sampling from a generic distribution. To this end, the authors in [26] employed Markov chain Monte Carlo sampling and Metropolis-Hastings random walk, to generate (dependent on a current state) and evaluate candidate points to compute their beliefs, and choose the best one. However, the performance of MPHL (both accuracy and complexity) is directly linked to the number of samples used.

The above RSS/AOA cooperative distributed localization algorithms are either excessively expensive computationwise [24], [26] or require knowledge about noise powers [25] and its distribution [26]; hence, they might not be convenient for practical use. Unlike these, a novel algorithm is proposed here based on a simple geometrical approach and multi-hopping, which has linear computational complexity and does not require any knowledge about noise powers nor 
its distribution. It can be summarized as follows. First, a Weighted Central Mass (WCM) approach is used to localize only sensors with direct links to some known reference points. Afterwards, the remaining sensors are localized by applying the law of cosines on (imaginary) triangles between sensors constructed by resorting to multi-hop approach, which offers straightforward interpretation of the problem as a Generalized Trust Region Sub-Problem (GTRS). Owing to some convenient properties, GTRS can be exactly solved by merely a bisection procedure. Finally, refinement step is performed on all location estimates by applying WCM and taking advantage of the current location estimates.

The remainder of this paper is structured as follows. Section II introduces the considered measurement models and formulates the target localization problem. In Section III. a detailed derivation of the proposed distributed approach is presented. Sections [V] and $\mathrm{V}$. validate the performance of the proposed algorithm in terms of computational complexity and localization accuracy, respectively. Lastly, Section VI summarizes the main findings of this work.

\section{PROBLEM Formulation}

Consider a sensor network in a 2-D space comprising a set of anchors (reference points), $|\mathcal{A}|=N$, (sensors whose locations are known) and a set of targets, $|\mathcal{T}|=M$ (sensors whose locations we desire to determine), where $|\bullet|$ represents the cardinality of a set. The true locations of sensors are denoted as $\boldsymbol{a}_{j} \in \mathbb{R}^{2}, \forall j \in \mathcal{A}$ and $\boldsymbol{x}_{i} \in \mathbb{R}^{2}, \forall i \in \mathcal{T}$, respectively. An edge between two sensors exists if and only if they are within the communication range, $R$, of each other. Hence, the sets of all target/anchor and target/target edges are respectively defined as $\mathcal{E}_{\mathcal{A}}=\left\{(i, j):\left\|\boldsymbol{x}_{i}-\boldsymbol{a}_{j}\right\| \leq R, \forall i \in \mathcal{T}, \forall j \in \mathcal{A}\right\}$ and $\mathcal{E}_{\mathcal{T}}=\left\{(i, j):\left\|\boldsymbol{x}_{i}-\boldsymbol{x}_{j}\right\| \leq R, \forall i, j \in \mathcal{T}, i \neq j\right\}$.

Throughout this work, it is assumed that all sensors can measure RSS quantity of the received signal, and that only anchors are appropriately equipped to measure AOA. The RSS sensed by the $i$-th sensor related to the transmission of the $j$-th sensor, is defined as [27, Ch. 3]

$$
P_{i j}=P_{0}-10 \gamma \log _{10} \frac{d_{i j}}{d_{0}}+n_{i j}, \forall(i, j) \in \mathcal{E}_{\mathcal{A}} \cup \mathcal{E}_{\mathcal{T}}, i \neq j,
$$

where $P_{0}$ is the RSS value measured at a short reference distance $d_{0}\left(d_{0} \leq d_{i j}\right), \gamma$ is the path loss exponent, $d_{i j}$ is the distance between sensors $i$ and $j$, and $n_{i j} \sim \mathcal{N}\left(0, \sigma_{n_{i j}}^{2}\right)$ is the log-normal shadowing term modeled as zero-mean Gaussian random variable with variance $\sigma_{n_{i j}}^{2}$. It is assumed that RSS observations between targets are symmetric.

By applying simple geometry, azimuth angle observations (at anchors) can be modeled respectively as [15]:

$$
\varphi_{i j}=\arctan \left(\frac{x_{i 2}-a_{j 2}}{x_{i 1}-a_{j 1}}\right)+m_{i j}, \forall(i, j) \in \mathcal{E}_{\mathcal{A}},
$$

where $s_{k l}$ denotes the $l$-th coordinate of $k$-th sensor, and $m_{i j}$ is the measurement error of the azimuth angle, modeled as a zero-mean von Mises random variable with the concentration parameter $\kappa_{m_{i j}} \in[0, \infty)$, i.e., $m_{i j} \sim V M\left(0, \kappa_{m_{i j}}\right)$. This distribution can be interpreted as a circular equivalent of the

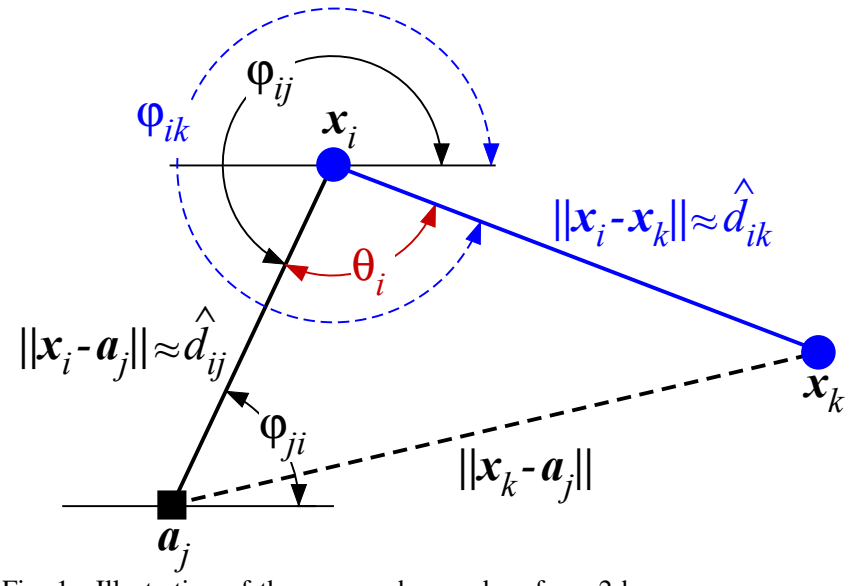

Fig. 1. Illustration of the proposed procedure for a 2-hop case.

Normal one, with a direct relation between the mean direction and the concentration parameter of the von Mises distribution and the mean and variance of the Normal one [28], [29].

For the sake of notation simplicity, define $\boldsymbol{P}=\left[P_{i j}\right]^{T}, \boldsymbol{\varphi}=$ $\left[\varphi_{i j}^{\mathcal{A}}\right]^{T}, \boldsymbol{X}=\left[\boldsymbol{x}_{1}, \ldots, \boldsymbol{x}_{M}\right],\left(\boldsymbol{X} \in \mathbb{R}^{2 \times M}\right)$, and $\mathcal{E}=\mathcal{E}_{\mathcal{A}} \cup \mathcal{E}_{\mathcal{T}}$. From (1), the conditional Probability Density Function (PDF) of RSS observations is given by

$$
f_{\boldsymbol{P}}(\boldsymbol{P} \mid \boldsymbol{X})=\prod_{(i, j) \in \mathcal{E}} \frac{1}{\sqrt{2 \pi \sigma_{n_{i j}}^{2}}} \mathrm{e}^{-\frac{\left(P_{i j}-P_{0}+10 \gamma \log _{10} \frac{d_{i j}}{d_{0}}\right)^{2}}{2 \sigma_{n_{i j}}^{2}}} .
$$

Similarly, from (2) the conditional PDF of an AOA observations can be written as

$$
f_{\boldsymbol{\varphi}}(\boldsymbol{\varphi} \mid \boldsymbol{X})=\prod_{(i, j) \in \mathcal{E}_{\mathcal{A}}} \frac{1}{2 \pi I_{0}\left(\kappa_{m_{i j}}\right)} \mathrm{e}^{\kappa_{m_{i j}} \boldsymbol{u}_{i j}^{T} \frac{\boldsymbol{a}_{j}-\boldsymbol{x}_{i}}{\left\|\boldsymbol{x}_{i}-\boldsymbol{a}_{j}\right\|}},
$$

where $I_{k}(\bullet)$ is the modified Bessel function of first kind of order $k$ [28], [29], and $\boldsymbol{u}_{i j}=\left[\cos \left(\varphi_{i j}\right), \sin \left(\varphi_{i j}\right)\right]^{T}$ is a unit vector.

A Maximum Likelihood (ML) estimator of $\boldsymbol{X}$ is obtained by maximizing (3) and (4). However, the ML problem is highly non-convex with no closed-form solution; thus, tackling it directly might not be feasible in practice.

\section{The Proposed Distributed APPROACH}

The distance that best estimates $\left\|\boldsymbol{x}_{i}-\hat{\boldsymbol{a}}_{j}\right\|$ in (3), with $\hat{\boldsymbol{a}}_{j}=$ $\boldsymbol{a}_{j}$, if $j \in \mathcal{A}$ and $\hat{\boldsymbol{a}}_{j}=\boldsymbol{x}_{j}$, if $j \in \mathcal{T}$, in the ML sense is

$$
\widehat{d}_{i j}=d_{0} 10^{\frac{P_{0}-P_{i j}}{10 \gamma}}, \forall(i, j) \in \mathcal{E} .
$$

Hence, it is pretty straightforward to obtain an estimate of $\boldsymbol{x}_{i}$ if it has an anchor neighbor, i.e.,

$$
\hat{\boldsymbol{x}}_{i}=\boldsymbol{a}_{j}+\widehat{d}_{i j} u_{i j},(i, j) \in \mathcal{E}_{\mathcal{A}} .
$$

However, due to the limited communication range of the sensors, only some targets will be able to directly communicate with anchors, but certainly not all of them. In such a case, one has to resort to alternative solutions, such as the proposed geometrical multi-hop approach, illustrated in Fig. 1

In Fig. 1, we are interested in determining the location of $\boldsymbol{x}_{k}$, which has a single target neighbor, $\boldsymbol{x}_{i}$, and a 2-hop anchor 
neighbor, $\boldsymbol{a}_{j}$ (there is no edge between $\boldsymbol{x}_{k}$ and $\boldsymbol{a}_{j}$ ). Note that an estimate of $\boldsymbol{x}_{i}$ can be obtained through (6), and that $\varphi_{i j}=$ $\varphi_{j i}+\pi$. Furthermore, since anchors exclusively can measure AOA, $\varphi_{i k}$ (and consequently $\theta_{i}$ ) is not known. Nevertheless, by applying the law of cosines to the (imaginary) triangle formed by the three sensors, one has that

$$
\left\|\boldsymbol{x}_{k}-\boldsymbol{a}_{j}\right\|^{2} \approx \widehat{d}_{i j}^{2}+\widehat{d}_{i k}^{2}-2 \widehat{d}_{i j} \widehat{d}_{i k} \cos \left(\theta_{i}\right) .
$$

Since $\cos \left(\theta_{i}\right)=\cos \left(\varphi_{i j}-\varphi_{i k}\right)=\boldsymbol{u}_{i j}^{T} \frac{\boldsymbol{x}_{k}-\boldsymbol{x}_{i}}{\left\|\boldsymbol{x}_{i}-\boldsymbol{x}_{k}\right\|}$, from (7) it follows that

$$
\left(2 \widehat{d}_{i j} \boldsymbol{u}_{i j}^{T}-2 \boldsymbol{a}_{j}^{T}\right) \boldsymbol{x}_{k}+\left\|\boldsymbol{x}_{k}\right\|^{2}+\left\|\boldsymbol{a}_{j}\right\|^{2}-\widehat{d}_{i j}^{2}-\widehat{d}_{i k}^{2}-2 \widehat{d}_{i j} \boldsymbol{u}_{i j}^{T} \boldsymbol{x}_{i} \approx 0,
$$

which can be written in vector form as

$$
\boldsymbol{A} \boldsymbol{y}_{k}-\boldsymbol{b} \approx \mathbf{0}
$$

where $\boldsymbol{A}=\left[2 \widehat{d}_{i j} \boldsymbol{u}_{i j}^{T}-2 \boldsymbol{a}_{j}^{T}, 1\right], \boldsymbol{y}_{k}=\left[\boldsymbol{x}_{k}^{T},\left\|\boldsymbol{x}_{k}\right\|^{2}\right]^{T}$, and $\boldsymbol{b}=\left[\widehat{d}_{i j}^{2}+\widehat{d}_{i k}^{2}+2 \widehat{d}_{i j} \boldsymbol{u}_{i j}^{T} \boldsymbol{x}_{i}-\left\|\boldsymbol{a}_{j}\right\|^{2}\right]$. By introducing an auxiliary variable $\alpha=\|\boldsymbol{x}\|^{2}$, from (8) one can derive a GTRS problem, i.e.,

$$
\underset{\boldsymbol{y}_{k}=\left[\boldsymbol{x}_{k}^{T}, \alpha\right]^{T}}{\operatorname{minimize}}\left\{\left\|\left(\boldsymbol{A} \boldsymbol{y}_{k}-\boldsymbol{b}\right)\right\|^{2}: \boldsymbol{y}_{k}^{T} \boldsymbol{D} \boldsymbol{y}_{k}+2 \boldsymbol{f}^{T} \boldsymbol{y}_{k}=0\right\},
$$

with

$$
\boldsymbol{D}=\left[\begin{array}{cc}
\boldsymbol{I}_{2} & \mathbf{0}_{2 \times 1} \\
\mathbf{0}_{1 \times 2} & 0
\end{array}\right], \boldsymbol{f}=\left[\begin{array}{c}
\mathbf{0}_{2 \times 1} \\
-1 / 2
\end{array}\right] .
$$

GTRS is characterized by minimizing a quadratic objective function over a quadratic constraint, and although non-convex in general, it is a monotonically decreasing function over an interval, which can readily be computed [10]. Therefore, GTRS is very suitable for solving via bisection procedure.

Based on the above procedures, we describe the proposed distributed approach in Algorithm 1, denoted as "WCM+GTRS". Basically, the algorithm can be divided into three phases. In the first phase, only targets with 1-hop anchor neighbors are localized based on a WCM approach of estimates obtained from (6) (as their weighted sum). Weights are introduced within the process in order to give more importance to nearby links. This line of reasoning is valid for both RSS and AOA measurements, as they are trusted more than the remote ones [8]. The localized targets then become quasianchors (or helpers [30]) for the following phases. Please note that for notation simplicity, we abuse strict formal notation at Lines $5,14,20$, where we use $\mathcal{S}(l)$ to denote the $l$-th element of the set $\mathcal{S}$, as if $\mathcal{S}$ were a vector. In the second phase, the remaining targets are localized by using the proposed multi-hop approach that leads to a GTRS framework. To do so, targets with most quasi-anchor neighbors are allowed to localize themselves first. Their direct neighbors play a role of pivots $\left(\boldsymbol{x}_{i}\right.$ in Fig. 1) and use their own 1-hop neighbors to form triangles which will allow derivation of (9) at the later stage 1 . Finally, a refinement of the estimates is performed by

\footnotetext{
${ }^{1}$ Since 2-D networks are considered here, the total of at least three 1-hop neighbors of the pivots are required to localize the target of interest. Pseudocode for this part was omitted from Algorithm 1 for the sake of simplicity, but if pivots do not have at least three 1-hop (localized) neighbors in total, they would resort to their (localized) 2-hop neighbors, and so on.
}

using WCM for each target without any specified order. Note that, in this process, all direct neighbors of each target are used, rather than just anchors.

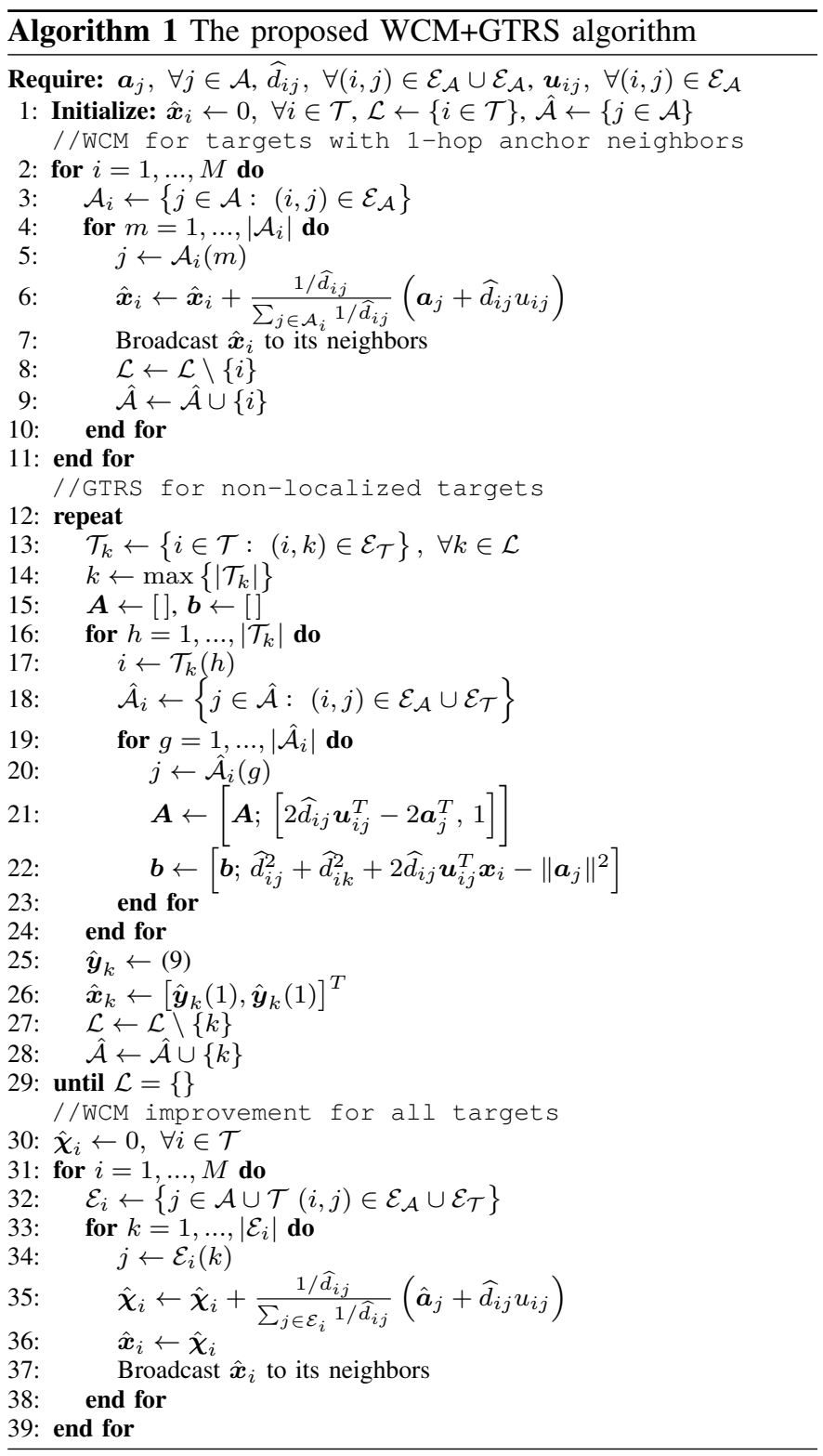

\section{Complexity Analysis}

To analyze the worst-case computational complexity, a fully connected network (i.e., the total number of edges for each target is $\left.\left|\mathcal{E}_{i}\right|=N+(M-1), \forall i \in \mathcal{T}\right)$ is considered and merely the dominating terms are considered, expressed as a function of $\left|\mathcal{E}_{i}\right|$. The results regarding computational complexity of the considered algorithms are summarized in Table I], where $S_{\max }$, $C_{\max }$, and $T_{\max }$ are used to denote the number of step in the bisection procedure in 1 , the number of generated candidates and the number of iterations in MPHL [26], respectively.

As expected, the Table I shows that SOCP is computationally the most intensive one. Even though MPHL has linear computational complexity in $\left|\mathcal{E}_{i}\right|$, due to high number of candidates that it requires in each generation step, its execution time is much higher than those of DLLS and WCM+GTRS. 
TABLE I

Complexity Analysis of the Considered Algorithms

\begin{tabular}{|c|c|}
\hline Algorithm & Complexity \\
\hline \hline WCM+GTRS & $\mathcal{O}\left(S_{\max } \times\left|\mathcal{E}_{i}\right|\right)$ \\
\hline SOCP & $\mathcal{O}\left(\left|\mathcal{E}_{i}\right|^{3.5}\right)$ \\
\hline DLLS & $\mathcal{O}\left(\left|\mathcal{E}_{i}\right|\right)$ \\
\hline MPHL & $\mathcal{O}\left(C_{\max } \times T_{\max } \times\left|\mathcal{E}_{i}\right|\right)$ \\
\hline
\end{tabular}

\section{Simulation Results}

This section presents a set of simulation results with the purpose of validating the performance of the proposed algorithm. Only connected networks are considered, where all sensors are deployed randomly inside a square with an edge length $B=30 \mathrm{~m}$ in each Monte Carlo $\left(M_{c}\right)$ run. The observations were generated through (1) and (2), with the reference distance set to $d_{0}=1 \mathrm{~m}$, the reference RSS to $P_{0}=20 \mathrm{dBm}$, and the PLE to $\gamma=3$. The number of steps in the bisection procedure was set to $S_{\max }=30$. Furthermore, $\sigma_{n_{i j}}=10 \mathrm{~dB}$ and $\kappa_{i j}=16.676$, which corresponds to the Gaussian standard deviation of $\sigma_{m_{i j}}=10 \mathrm{deg}$, since $\sigma_{m_{i j}}^{2}=1-I_{1}\left(\kappa_{i j}\right) / I_{0}\left(\kappa_{i j}\right)$ [29]. The employed performance metric is the Averaged Root Mean Square Error (ARMSE), ARMSE $=\sqrt{\sum_{j=1}^{M_{c}} \sum_{i=1}^{M} \frac{\left\|\boldsymbol{x}_{i j}-\widehat{\boldsymbol{x}}_{i j}\right\|^{2}}{M M_{c}}}$, where $\widehat{\boldsymbol{x}}_{i j}$ is the estimate of the true target location, $\boldsymbol{x}_{i j}$, in the $j$-th $M_{c}$ run. The new method is compared with SOCP [24], DLLS [25] and MPHL [26] algorithms ${ }^{2}$ It is worth mentioning that, unlike the new method and SOCP, DLLS and MPHL assume the AOA quantity is measured by all sensors, rather than just anchors.

Fig. 2 illustrates one particular output by WCM+GTRS for the network shown in Fig. 22. Firstly, all targets with direct anchor neighbors are localized by WCM, Fig. 2p. The localized targets are then used as quasi-anchors in order to localize the remaining targets Fig. 2r. Finally, the refinement step is performed for all targets, Fig. 2 2 d. It can be seen from the figure that the overall accuracy is fairly good in general, and that even the targets with no direct anchor neighbors (e.g., the four far-left top ones) can be localized fairly accurately.

Fig. 3 illustrates ARMSE (m) versus $N$ performance comparison. Naturally, the figure shows that all algorithms benefit from adding more reference points into the network. This behaviour is more evident for the new method and SOCP, while DLLS gets quickly saturated, and only marginally improves its performance. Even though DLLS (with perfect knowledge about noise powers) shows the best performance for all considered $N$, when it exploits defective information $\left(\hat{\sigma}_{i j}=15(\mathrm{~dB}, \mathrm{deg})\right)$ its performance deteriorates drastically. Nonetheless, even when it uses perfect knowledge about noise powers (as well as measurements of the AOA quantity at targets), its performance is practically matched by the new method for large $N$.

Fig. 4 illustrates ARMSE (m) versus $M$ performance comparison. It is noticeable that, while the performance of other

\footnotetext{
${ }^{2}$ DLLS and MPHL assume perfect knowledge of the noise powers, which might not be feasible in practice. Hence, in Figs. 3,4 and 5 we also implemented DLLS with imperfect knowledge about the noise powers, just to give an intuition to the reader how this imperfection could affect its accuracy.
}

methods is maintained or exacerbated with the increase of $M$, the performance of the proposed one actually betters when more targets are introduced in the network. This can be explained to some extent by the fact that as the network becomes denser, it is more likely to acquire more pivots, which obviously favors the proposed geometric approach.

Fig. 5 illustrates ARMSE (m) versus $R(\mathrm{~m})$ performance comparison. One can notice a somewhat unexpected behavior of most of the considered algorithms (actually all with linear computational complexity), since their performance does not better as $R$ increases. This result suggests that perhaps potentially increased quantity of information is not benignant by default, and that there might be some highly corrupted edges in the network that would be preferable to omit in the localization process, since they might harm the overall performance. The only algorithm that seems to be robust to this phenomenon is SOCP, which might be explained to some extent by the fact that SOCP has considerably higher computational complexity than the remaining ones. Nevertheless, the figure indicates that the proposed method betters from the increased information until a certain point, after which its performance mildly deteriorates, but is still very close to that of SOCP and MPHL.

Finally, Fig. 6 illustrates ARMSE (m) versus $N$ performance comparison of the proposed algorithm when different number of hops are used in the refinement step, i.e., up to 2,3 , and 4 hops. The figure shows that by using information from multi-hop neighbors one can improve significantly the performance of the proposed algorithm. However, it seems that WCM+GTRS gets saturated fairly quickly with the additional information, ans using more than two-hops does not bring any benefit. In fact, its performance degrades marginally as more than two-hops are employed. This can be explained to some extent by the fact that the additional information used from multi-hop neighbors is estimated, rather than measured as in the case of direct neighbors; hence, exploiting fairly distant neighbors actually propagates the estimation error. Nevertheless, this deterioration is only marginal in comparison with using up to two-hop neighbors, and still offers considerable advantage over the original WCM+GTRS.

\section{CONCLUSiOnS}

This work presented a novel geometric algorithm for target localization in distributed networks, in which all sensors are capable of acquiring RSS measurements, but only anchors are assumed suitably equipped to measure AOA. The proposed approach can be explained in three parts. Firstly, only the targets with direct edges to anchors are localized by using the WCM approach applied to a set of candidate points, obtained in a simple geometric manner by exploiting range estimates (from RSS) and AOA measurements. These targets are then treated as quasi-anchors. Secondly, the remaining targets are localized by applying the law of cosines on imaginary triangles formed by targets' direct and indirect neighbors, leading to the GTRS. Finally, refinement of the estimated locations of all targets is performed by applying WCM. Unlike some existing methods, the proposed one does not require all sensors to measure AOA 


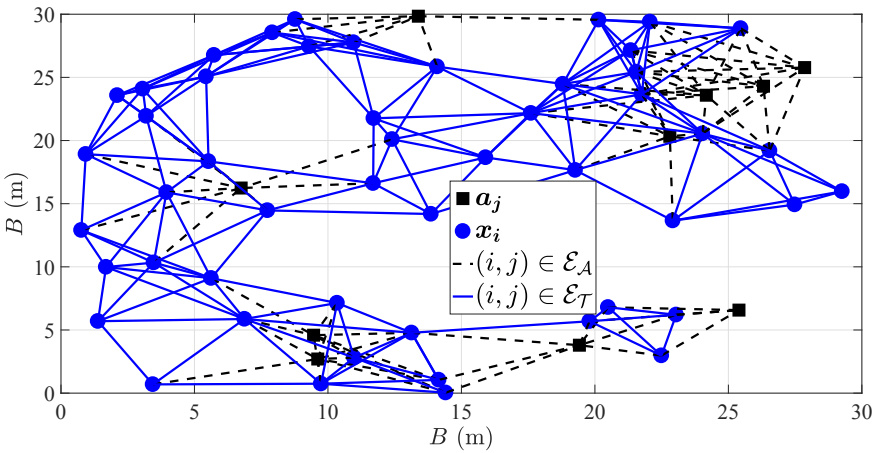

(a) Network example

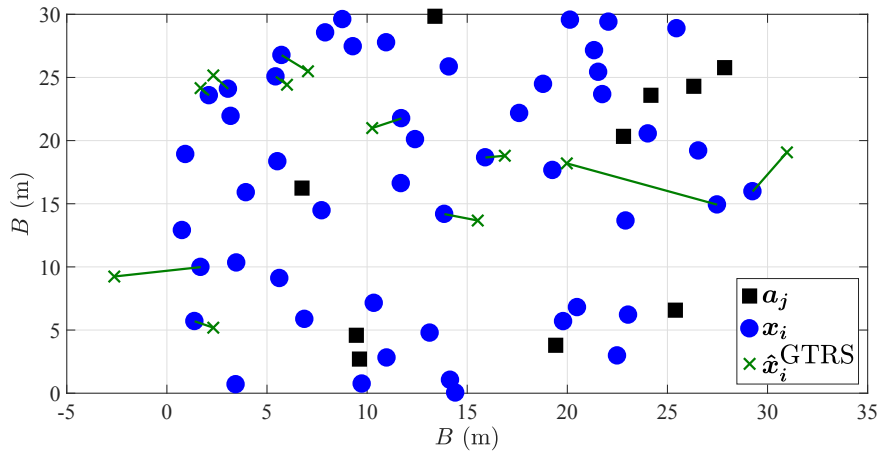

(c) Estimation via GTRS

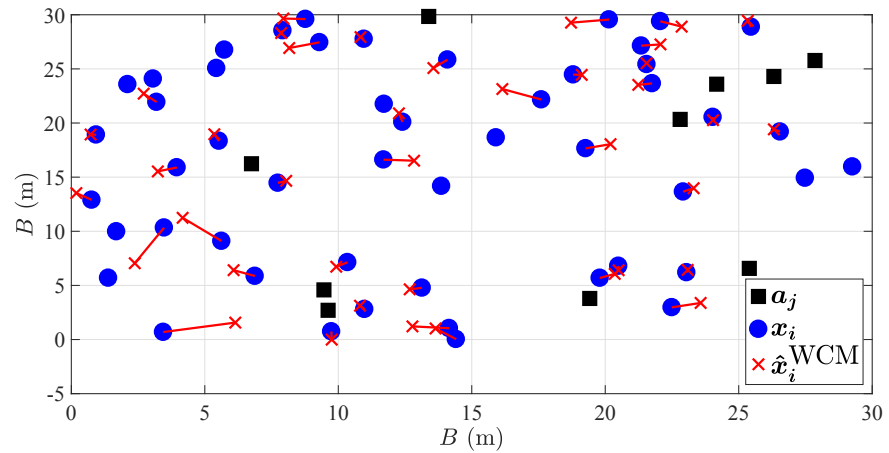

(b) Estimation via WCM

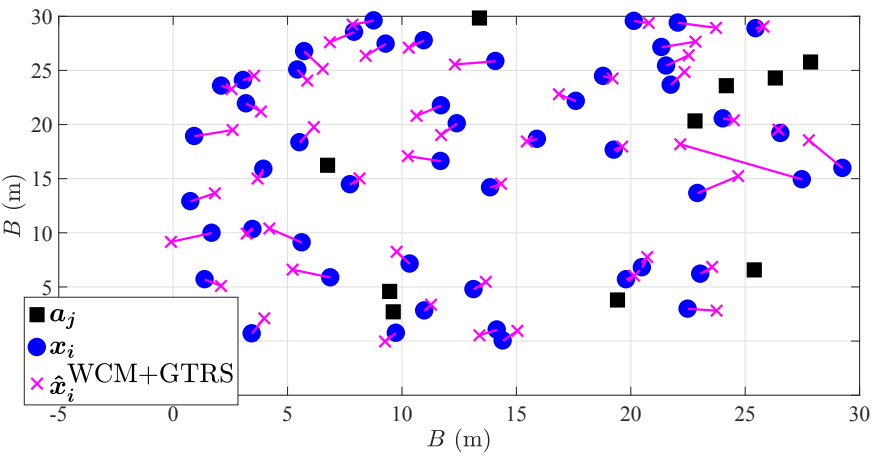

(d) Estimation via WCM+GTRS

Fig. 2. Illustration of the estimation process by using the proposed WCM+GTRS algorithm for $N=10, M=50$ and $R=7 \mathrm{~m}$.

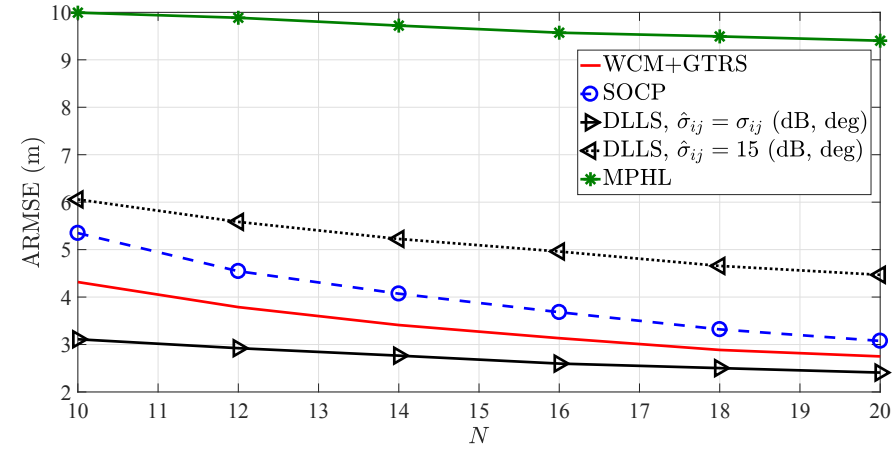

Fig. 3. ARMSE (m) versus $N: M=50, R=10 \mathrm{~m}, M_{c}=1000$.

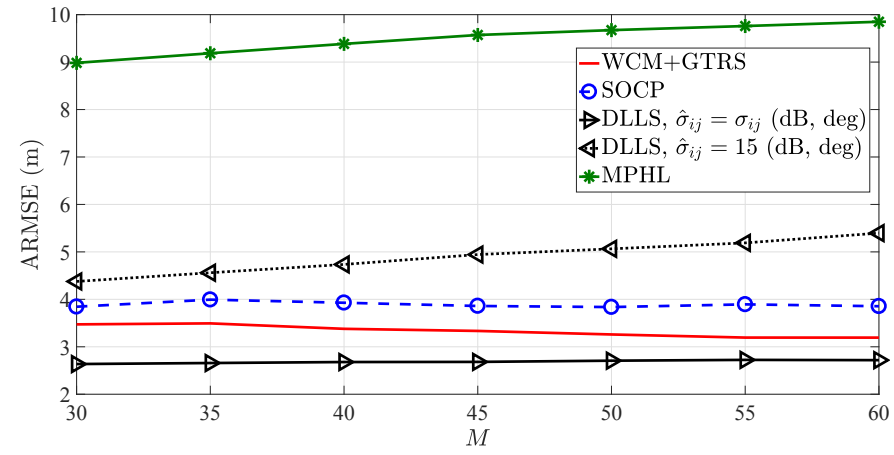

Fig. 4. ARMSE (m) versus $M: N=15, R=10 \mathrm{~m}, M_{c}=1000$.

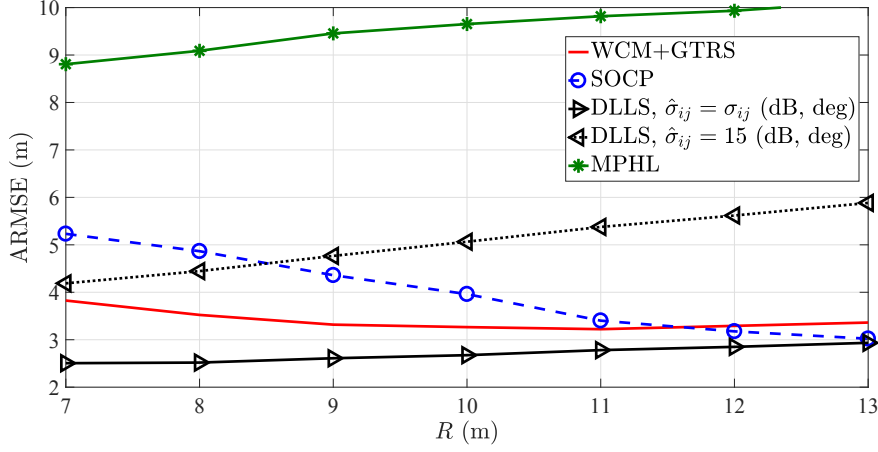

Fig. 5. ARMSE (m) versus $R(\mathrm{~m}): N=15, M=50, M_{c}=1000$.

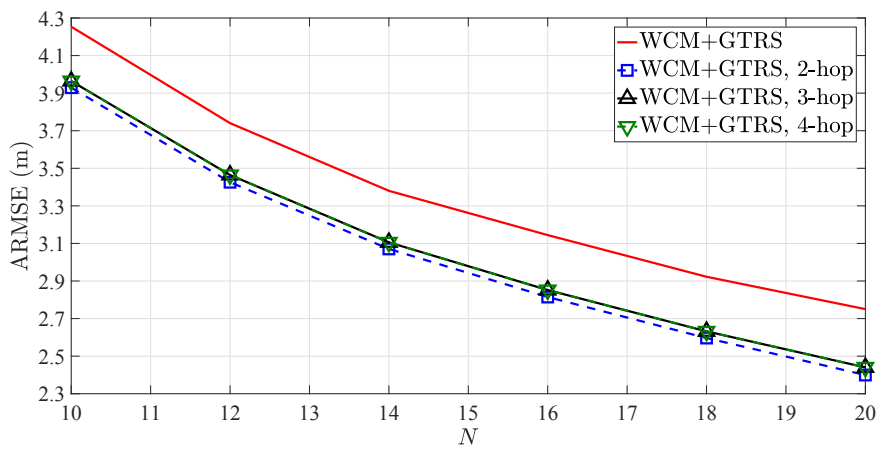

Fig. 6. ARMSE (m) versus $N: M=50, R=10 \mathrm{~m}, M_{c}=1000$. 
quantity (which can significantly reduce the network costs) nor to have information about the noise powers a priori. Even so, it showed good performance in all considered scenarios, where it outperformed some computationally more demanding approaches (SOCP and MPHL), and was competitive with DLLS that takes advantage of certain assumptions (which might not hold in practice) to enhance its performance.

\section{ACKNOWLEDGEMENT}

This work was partially supported by Fundação para a Ciência e a Tecnologia under Projects UID/MULTI/04111/2019, UID/EEA/00066/2019, foRESTER PCIF/SSI/0102/2017, and IF/00325/2015.

\section{REFERENCES}

[1] K. Witrisal and C. Antn-Haro "Whitepaper on New Localization Methods for 5G Wireless Systems and the Internet-of-Things," COST CA15104 (IRACON), April 2018.

[2] S. Safavi, U. A. Khan, S. Kar and J. M. F. Moura, "Distributed Localization: A Linear Theory," Proceedings of the IEEE, vol. 106, no. 7, pp. 1204-1223, July 2018.

[3] D. Pedro, S. Tomic, L. Bernardo, M. Beko, R. Oliveira, R. Dinis, P. Pinto, and P. Amaral, "Algorithms for Estimating the Location of Remote Nodes Using Smartphones," IEEE Access, vol. 17, pp. 33713-33727, March 2019.

[4] S. Tomic, M. Beko, and R. Dinis, "RSS-based Localization in Wireless Sensor Networks Using Convex Relaxation: Noncooperative and Cooperative Schemes," IEEE Trans. Veh. Technol., vol. 64, no. 5, pp. 20372050, May 2015.

[5] A. Coluccia and A. Fascista, "On the Hybrid TOA/RSS Range Estimation in Wireless Sensor Networks," IEEE Trans. Wirel. Commun. vol. 17, no. 1, pp. 361-371, January 2018.

[6] S. Tomic, M. Beko, and R. Dinis, "Distributed RSS-Based Localization in Wireless Sensor Networks Based on Second-Order Cone Programming," Sensors, vol. 14, no. 10, pp. 18410-18432, Oct. 2014.

[7] L. Gazzah, L. Najjar, and H. Besbes, "Hybrid RSS/AOA Hypothesis Test for NLOS/LOS Base Station Discrimination and Location Error Mitigation," Trans. Emerging Telecomm. Technol., vol. 27, no. 5, pp. 629-639, May 2016.

[8] S. Tomic, M. Beko, R. Dinis, and P. Montezuma, "A Closed-Form Solution for RSS/AoA Target Localization by Spherical Coordinates Conversion," IEEE Wirel. Commun. Lett., vol. 5, no. 6, pp. 680-683, December 2016

[9] A. Tahat, G. Kaddoum, S. Yousefi, S. Valaee, and F. Gagnon, "A Look at the Recent Wireless Positioning Techniques With a Focus on Algorithms for Moving Receivers," IEEE Access, vol. 2016, no. 4, pp. 6652-6680, November 2016.

[10] S. Tomic and M. Beko, "A Robust NLOS Bias Mitigation Technique for RSS-TOA-Based Target Localization," IEEE Sign. Process. Letters, vol. 26, no. 1, pp. 64-68, January 2019.

[11] M. W. Khan, N. Salman, A. H. Kemp, and L. Mihaylova, "Localisation of Sensor Nodes with Hybrid Measurements in Wireless Sensor Networks," Sensors, vol. 16, no. 7, pp. 1-16, July 2016.

[12] S. Tomic, M. Beko and R. Dinis, "3-D Target Localization in Wireless Sensor Network Using RSS and AoA Measurement," IEEE Trans. Vehic. Technol., vol. 66, no. 4, pp. 3197-3210, April 2017.

[13] P. Biswas, H. Aghajan, and Y. Ye, "Semidefinite Programming Algorithms for Sensor Network Localization Using Angle of Arrival Information," Asilomar, pp. 220-224, October 2005.

[14] S. Tomic and M. Beko, "A Bisection-based Approach for Exact Target Localization in NLOS Environments," Sign. Process., vol. 143, pp. 328335, February 2018.

[15] K. Yu, "3-D Localization Error Analysis in Wireless Networks," IEEE Trans. Wirel. Commun., vol. 6, no. 10, pp. 3473-3481, October 2007.

[16] S. Tomic, M. Beko, R. Dinis, and P. Montezuma, "Distributed Algorithm for Target Localization in Wireless Sensor Networks Using RSS and AoA Measurements," Pervasive and Mobile Computing, vol. 37, pp. 6377, October 2016.

[17] S. Wang, B. R. Jackson, and R. Inkol, "Hybrid RSS/AOA Emitter Location Estimation Based on Least Squares and Maximum Likelihood Criteria," IEEE QBSC, pp. 24-29, June 2012.
[18] S. Tomic, M. Beko, and M. Tuba, "A Linear Estimator for Network Localization Using Integrated RSS and AOA Measurements," IEEE Sign. Process. Letters, vol. 26, no. 3, pp. 405-409, March 2019.

[19] Y. Wang and K. C. Ho, "An Asymptotically Efficient Estimator in Closed-Form for 3D AOA Localization Using a Sensor Network," IEEE Trans. Wirel. Commun., vol. 14, no. 12, pp. 6524-6535, December 2015.

[20] R. Parker and S. Valaee, "Vehicular Node Localization Using Received Signal-Strength Indicator," IEEE Trans. Vehic. Technol., vol. 56, no. 6 , pp. 3371-3380, November 2007.

[21] I. Bisio, F. Lavagetto, M. Marchese, and A. Sciarrone, "Smart Probabilistic Fingerprinting for WiFi-based Indoor Positioning with Mobile Devices," Pervasive and Mobile Computing, vol. 31, pp. 107-123, September 2016.

[22] A. Fascista, G. Ciccarese, A. Coluccia, and G. Ricci, "Angle of ArrivalBased Cooperative Positioning for Smart Vehicles," IEEE Trans. Intell. Transport. Syst., vol. 19, no. 9, pp. 2880-2892, September 2018.

[23] I. Bisio, C. Garibotto, F. Lavagetto, and A. Sciarrone, "Outdoor Places of Interest Recognition Using WiFi Fingerprints," IEEE Trans. Vehic. Technol., vol. 68, no. 5, pp. 5076-5086, May 2019.

[24] S. Tomic, M. Beko, and R. Dinis, "Distributed RSS-AoA Based Localization with Unknown Transmit Powers," IEEE Wirel. Commun. Lett., vol. 5, no. 4, pp. 392-395, August 2016

[25] M. W. Khan, N. Salman, and A. H. Kemp, "Optimised Hybrid Localisation with Cooperation in Wireless Sensor Networks," IET Sign. Process., vol. 11, no. 3, pp. 341-348, May 2017.

[26] H. Naseri and V. Koivunen, "A Bayesian Algorithm for Distributed Network Localization Using Distance and Direction Data," IEEE Trans. Sign. Inform. Process. Networks, vol. 5, no. 2, pp. 290-304, June 2019.

[27] T. S. Rappaport. Wireless Communications: Principles and Practice. Prentice-Hall: Upper Saddle River, NJ, USA, 1996.

[28] K. V. Mardia. Statistics of Directional Data, Academic Press, Inc.: 24/28 Oval Road, London, England, 1972.

[29] C. Forbes, M. E. Evans, N. Hastings, and B. Peacock. Statistical Distributions, Fourth Edition, John Wiley \& Sons, Inc.: Hoboken, NJ, USA, 2011.

[30] A. Coluccia, F. Ricciato, and G. Ricci, "Positioning Based on Signals of Opportunity," IEEE Commun. Letters, vol. 18, no. 2, pp. 356-359, February 2014.

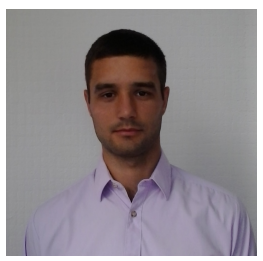

Slavisa Tomic received the M.S. degree in traffic engineering according to the postal traffic and telecommunications study program from University of Novi Sad, Serbia in 2010, and the $\mathrm{PhD}$ degree in electrical and computer engineering from University Nova of Lisbon, Portugal in 2017. He is currently an Assistant Professor at the Universidade Lusófona de Humanidades e Tecnologias, Lisbon, Portugal. His research interests include target localization in wireless sensor networks, and non-linear and convex optimization.

Marko Beko was born in Belgrade,

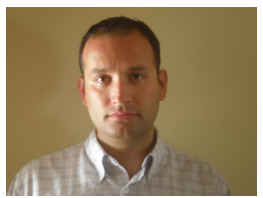
Serbia, on November 11, 1977. He received the $\mathrm{PhD}$ degree in electrical and computer engineering from Instituto $\mathrm{Su}$ perior Tecnico, Lisbon, Portugal, in 2008. $\mathrm{He}$ received the title of "Professor com Agregação" in Electrical and Computer Engineering from Universidade Nova de Lisboa, Lisbon, Portugal, in 2018. Currently, he is a Full Professor (Professor Catedrático) at the Universidade Lusófona de Humanidades e Tecnologias, Lisbon, Portugal. He serves as an Associate Editor for the IEEE Open Journal of the Communications Society and Elsevier Journal on Physical Communication. He is the winner of the 2008 IBM Portugal Scientific Award. 